\title{
2M0415+0154: a new bright, short-period subdwarf B pulsator ${ }^{\star}$
}

\author{
R. Oreiro ${ }^{1}$, R. H. Østensen ${ }^{1}$, E. M. Green ${ }^{2}$, and S. Geier ${ }^{3}$ \\ ${ }^{1}$ Instituut voor Sterrenkunde, K. U. Leuven, Celestijnenlaan 200D, 3001 Leuven, Belgium \\ e-mail: [raquel; roy]@ster. kuleuven.be \\ 2 Steward Observatory, University of Arizona, Tucson, AZ 85721, USA \\ e-mail: bgreen@as.arizona.edu \\ 3 Dr. Remeis-Sternwarte, Sternwartstr. 7, 96049 Bamberg, Germany \\ e-mail: geier@sternwarte.uni-erlangen.de
}

Received 21 November 2008 / Accepted 28 January 2009

\begin{abstract}
Context. The discovery of stellar oscillations in some subdwarf B stars presents the opportunity of studying the interior structure of these objects, thereby clarifying their possible formation channels.

Aims. Our ongoing survey attempts to increase the number of pulsating hot subdwarfs to better understand the complexities of the various subgroups within the population of pulsators and to identify targets suitable for seismological analysis.

Methods. Light curves for a list of potential pulsators were obtained at the Nordic Optical Telescope. The candidates were mainly selected based on similar $T_{\text {eff }}$ and $\log g$ as known pulsators. A frequency analysis of the light curves was performed to investigate the multiperiodic photometric variability.

Results. From the sample we surveyed in 2007, 2M0415+0154 was the only star clearly showing photometric modulations. Followup photometry revealed three clear pulsation modes with periods between 143 and $150 \mathrm{~s}$. Additional spectroscopic data was taken on $2 \mathrm{M} 0415+0154$ to refine its physical parameters. We find $T_{\text {eff }}=34000 \pm 500 \mathrm{~K}, \log g=5.80 \pm 0.05 \operatorname{dex}, \log \left(N_{\mathrm{He}} / N_{\mathrm{H}}\right)=-1.60 \pm 0.05$, which are typical of sdB pulsators in this period range. No evidence of a companion was found.

Conclusions. A new short-period sdB star has been discovered, 2M0415+0154. Its physical parameters and oscillation frequencies agree with those of other V361 Hya stars. The relative brightness and simple frequency spectrum of this star make it a suitable target for empirical mode identification, essential for a seismological analysis of simple pulsators.
\end{abstract}

Key words. stars: oscillations - stars: subdwarfs - stars: fundamental parameters - stars: individual: 2M0415+0154

\section{Introduction}

Asteroseismology has been proven to be a powerful method to explore stellar interiors, including that of our own Sun (e.g. Winget \& Kepler 2008; Christensen-Dalsgaard 2002). Information on global stellar parameters, but also on the internal structure can be derived from a star undergoing stellar oscillations. The internal stratification is inaccessible by any other means and can be used to establish the evolutionary status of individual stars and of a particular class of pulsators in general. Asteroseismology has thus the potential to refine the theory of stellar evolution by studying the different groups of pulsating stars all across the Hertzsprung-Russell diagram.

Hot subdwarfs are evolved objects that have already completed their main sequence and red giant phases (Heber 1986). Theoretical evolutionary sequences for the extreme horizontal branch (EHB) reproduce the position of B-type hot subdwarfs (sdBs) in a $\log g-T_{\text {eff }}$ diagram (Dorman et al. 1993). Such models consist of helium core-burning stars with a $\sim 0.5 M_{\odot}$ canonical mass. Their hydrogen dominated envelopes are too thin to ignite $\mathrm{H}$-shell burning, and their evolution will take them directly to the white dwarf (WD) cooling track, bypassing the asymptotic giant branch and planetary nebulae stages. After core helium exhaustion, sdB evolutionary tracks cross the domain of the O-type hot subdwarfs (sdOs), implying that post-EHB stars may explain

* Based on observations from the Nordic Optical Telescope. some of the sdOs. However, the identification of different subgroups in the $\mathrm{sdO}$ domain requires a more complicated picture, with additional formation channels needed to account for the complete population (Stroeer et al. 2007; Green et al. 2008).

Exactly how the hot subdwarfs can lose most of their envelope, and still start He-core burning, is yet to be answered. Enhanced mass loss during the red giant stage has been proposed (Dorman et al. 1993), but also a variety of binary interaction channels (Han et al. 2002, 2003), based on the observational result that a high fraction of hot subdwarfs appear to be in a binary system (Green et al. 1997; Maxted et al. 2001; Napiwotzki et al. 2004a).

Fortunately, some hot subdwarfs exhibit stellar oscillations, allowing seismological tools to be applied. In particular, two types of pulsating sdBs have been identified. V361 Hya stars consist of sdBs with short period photometric modulations (2-10 min), explained as low order $(n)$, low degree $(\ell) p$-modes (Kilkenny et al. 1997; Charpinet et al. 1997), which coexist with sdBs not observed to vary at the high end of the temperature range for these stars. At the cooler end, a much larger fraction of sdBs seem to be pulsating, but with longer periods $(\sim 1 \mathrm{~h})$ and low amplitudes of a few millimagnitudes at best (Green et al. 2003). These pulsators are now officially named V1093 Her stars, after the prototype. Their oscillations are understood in terms of $g$-modes of high $n$ excited by the same $\kappa$ mechanism as for V361 Hya stars (Fontaine et al. 2003; Jeffery \& Saio 2006). 
Table 1. Log of photometric and spectroscopic observations. The filter and the cycle time $(\Delta t)$ is indicated for the photometric runs.

\begin{tabular}{cccccclll}
\hline \hline Date & UT start & Length & $\Delta t[\mathrm{~s}]$ & Filter & Passband $[\AA]$ & Telescope & Instrument & Observer \\
\hline $2004-01-28$ & $05: 07: 43$ & $1000 \mathrm{~s}$ & - & - & $3620-6895$ & Bok & B\&C & BG \\
$2007-10-24$ & $02: 15: 00$ & $02^{\mathrm{h}} 13^{\mathrm{m}}$ & 15 & $W$ & $4000-7150$ & NOT & ALFOSC & RO \\
$2007-12-15$ & $01: 36: 25$ & $02^{\mathrm{h}} 07^{\mathrm{m}}$ & 20 & $B$ & $3600-5200$ & NOT & ALFOSC & RØ \\
$2007-12-15$ & $03: 19: 32$ & $05^{\mathrm{h}} 29^{\mathrm{m}}$ & 34 & $B$ & $3600-5200$ & Kuiper & MONT4K & BG \\
$2007-12-16$ & $23: 23: 16$ & $02^{\mathrm{h}} 20^{\mathrm{m}}$ & 20 & $B$ & $3600-5200$ & NOT & ALFOSC & RØ \\
$2007-12-30$ & $06: 16: 04$ & $1200 \mathrm{~s}$ & - & - & $3620-6895$ & Bok & B\&C & BG \\
$2007-12-30$ & $06: 42: 10$ & $1125 \mathrm{~s}$ & - & - & $3620-6895$ & Bok & B\&C & BG \\
$2007-12-31$ & $06: 24: 36$ & $950 \mathrm{~s}$ & - & - & $3620-6895$ & Bok & B\&C & BG \\
$2008-01-01$ & $05: 46: 06$ & $1100 \mathrm{~s}$ & - & - & $3620-6895$ & Bok & B\&C & BG \\
$2008-01-12$ & $02: 58: 48$ & $200 \mathrm{~s}$ & - & - & $3880-4380$ & ESO/NTT & EMMI & SG \\
$2008-01-13$ & $02: 52: 15$ & $200 \mathrm{~s}$ & - & - & $3880-4380$ & ESO/NTT & EMMI & SG \\
\hline
\end{tabular}

Moreover, a few sdBs have been discovered showing simultaneously short and long period oscillations (both $p$ - and $g$-modes Schuh et al. 2006). Interestingly, all these stars lie just on the temperature boundary between the V361 Hya and V1093 Her objects. Another pulsator, located in the hot end of the sdB instability region, stands out as it is exceptionally helium rich (a He-sdB star, LS IV-14 ${ }^{\circ} 116$ Ahmad \& Jeffery 2005), and has periods more typical for the V1093 Her stars than the V361 Hya stars which surrounds it in the $T_{\text {eff }} / \log g$ plane. Furthermore, one single sdO has been recently discovered to be a very rapid multimode pulsator (Woudt et al. 2006). Observational searches for more sdO pulsators are ongoing, but evidence so far indicates that pulsations among these stars are rare (Rodríguez-López et al. 2007).

In light of the many different types of pulsators among the hot subdwarfs, many of which must have evolved into the subdwarf stage along different evolutionary paths, seismological studies have a particular potential. Differences in internal structure left behind by differing evolutionary channels can produce observable differences in the pulsation spectra. As asteroseismic models improve in sophistication and precision, the evolutionary paths can actually be distinguished by asteroseismology, as demonstrated recently by $\mathrm{Hu}$ et al. (2008).

Within this context, our group is involved in a long-term campaign to detect new pulsating sdBs, specially those of the V361 Hya type, taking advantage of our optimized observing technique to resolve short period variabilities (Østensen et al. 2001). Here we report the discovery of periodic modulations in the light curve of one such star. The range of periods and the physical parameters derived for this star clearly identifies it as a new member of the V361 Hya pulsators. This brings the number of confirmed short period pulsators up to 40 .

The object presented here was taken from a sample of blue stars selected by 2MASS colors (Skrutskie et al. 2006), for which there is an ongoing program using the $\mathrm{B} \& \mathrm{C}$ spectrograph on the University of Arizona/Steward Observatory $2.3 \mathrm{~m}$ Bok telescope on Kitt Peak, to obtain high $S / N$ spectra for a complete, magnitude-limited sample of hot subdwarfs with $V \leq 14.5$. Winter et al. (2006) analyzed 84 spectra from this sample as part of a $\mathrm{Ph}$.D. work on automatic analysis of stellar spectra, and the physical parameters he determined were incorporated into our list of likely pulsator candidates. The full IAU designation of our target is 2MASS J04155016+0154209, but we will refer to it hereafter as $2 \mathrm{M} 0415+0154$ for short. The 2MASs photometry is $J=14.513, H=14.627, K=14.656$, and the NOMAD cata$\log$ (Naval Observatory Merged Astrometric Dataset, Zacharias et al. 2005) provides the photographic magnitudes, $B=13.61$, $V=13.79, R=14.34$, where the $B$ and $V$ measurements

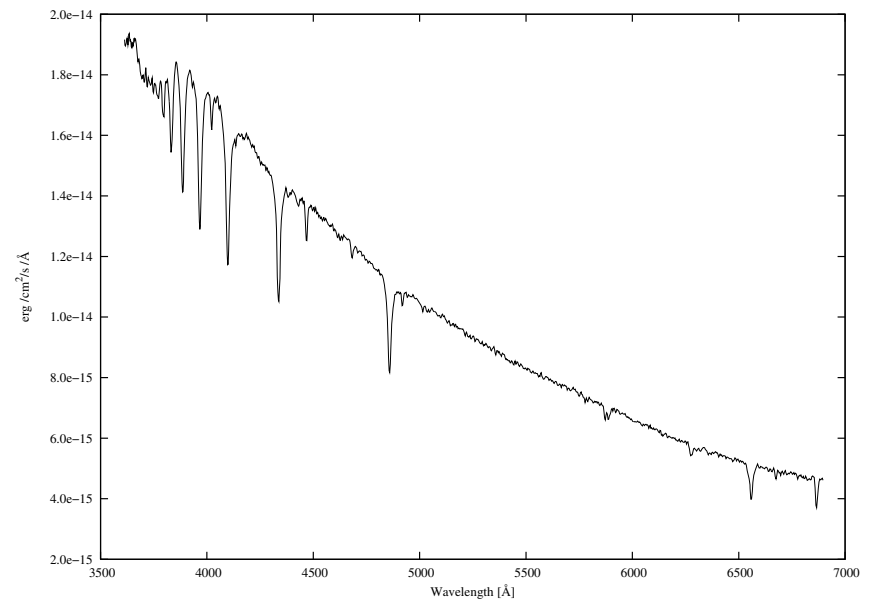

Fig. 1. The flux calibrated discovery spectrum of 2M0415+0154.

originates from the YB6 catalog and the $R$ measurement from the USNO-B1.0 catalog. The NOMAD catalog also lists proper motion measurements, but they are well within the 3-sigma error of $18.6 \mathrm{mas} / \mathrm{yr}$. The GALEX satellite has measured the $U V$ flux of this object, and finds the $U V$ magnitudes for the far $U V$ (FUV: 1350-1780 $\AA$ ) and near $U V(N U V: 1770-2730 \AA)$ bands to be $F U V=13.072$ and $N U V=13.499$.

In the following sections we will present our discovery data that reveals this object to be a fast pulsator, as well as follow-up photometric and spectroscopic observations.

\section{Observations}

2M0415+0154 was first observed during a three night run at the $2.5 \mathrm{~m}$ Nordic Optical Telescope (NOT) on La Palma, Canary Islands (Spain), as part of our campaign to search for new short period pulsating hot subdwarf stars. Follow-up observations were obtained a few months later, as well as follow-up spectroscopy to explore its binary status. A log of all our observations is shown in Table 1.

\subsection{Fast photometry}

The Andalucia Faint Object Spectrograph and Camera (ALFOSC) allows operation in windowed mode, where only small regions around target and reference stars (windows) are sampled on the CCD. After selecting the target, an area free of stars to determine the sky background, and typically 3-4 reference stars, readout times of $\sim 4 \mathrm{~s}$ are obtained, compared to $80-90 \mathrm{~s}$ for reading a 


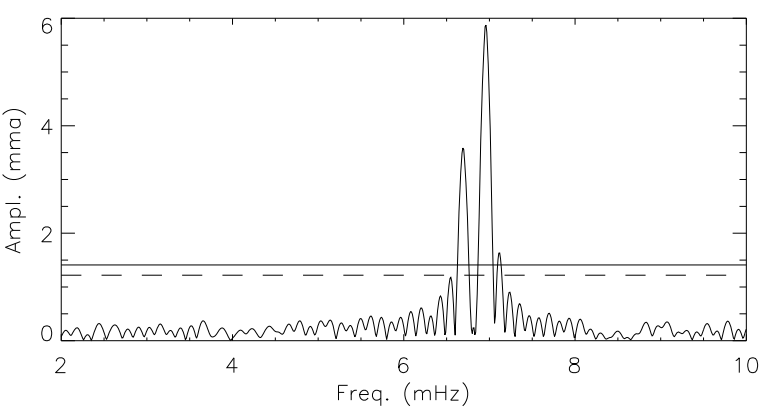

Fig. 2. Amplitude spectrum of $2 \mathrm{M} 0415+0154$ obtained from the discovery run. Continuous (broken) horizontal lines indicate 3.7 (3.2) times the noise level, as an indication of $99 \%$ (90\%) of significance level (Alvarez et al. 1998).

full frame. Efficient fast photometry is thus easily achieved with this mode, as required for periods as short as $60 \mathrm{~s}$ to be detected. For $2 \mathrm{M} 0415+0154$ we chose a cycle time of 15 or $20 \mathrm{~s}$, which includes 11 or $16 \mathrm{~s}$ of integration and a $4 \mathrm{~s}$ readout time.

The Real Time Photometry package (Østensen \& Solheim 2000) was used to analyse the data. It is specially designed to deal with windowed formats, and includes standard reduction steps. It also produces light curves and amplitude spectra automatically as images are being acquired. This is particularly useful to discern in real time whether a star is pulsating or not.

From our candidates surveyed during the 2007 observing season, only 2M0415+0154 showed clear signs of variability.

The star was observed for $2^{\mathrm{h}} 13^{\mathrm{m}}$ with a special wide filter that effectively encompasses all wavelengths from $B$ to $R$ with more than $90 \%$ transmission (NOT \#92) ${ }^{1}$. This filter (which we refer to as the $W$ filter) has the same center as that of the $V$-band (5500 $\AA$ ), but is a full $2750 \AA$ wide. The amplitude spectrum obtained is shown in Fig. 2. Two peaks are clearly well above the significance threshold, indicated as a continuous horizontal line in the figure, demonstrating that $2 \mathrm{M} 0415+0154$ is a new multiperiodic variable.

Soon after this discovery, 2M0415+0154 was reobserved at the NOT two nights in December, 2007. A $5^{\mathrm{h}} 29^{\mathrm{m}}$ run was also obtained at the Steward Observatory $1.2 \mathrm{~m}$ Kuiper Telescope on Mt. Bigelow, which slightly overlaps with the first night at NOT (see Table 1). It was acquired using the Montréal 4K CCD camera, which is a joint venture between the University of Arizona and the Université de Montréal ${ }^{2}$. The blue sensitive CCD has a dual amplifier readout, optimized to reduce overhead times for time-series photometry while maintaining high precision, and covers an area of $9.7^{\prime} \times 9.7^{\prime}$, allowing a good choice of reference stars over most of the sky.

This time the star was observed in the $B$-band at both telescopes, in order to obtain comparable photometric amplitudes in the two datasets. The integration time for the NOT runs was $15.2 \mathrm{~s}$ with a cycle time of 20.0 s, and for the Kuiper Telescope run the integration time was $22.0 \mathrm{~s}$ with a cycle time of $34 \mathrm{~s}$. The light curve of this object is shown in Fig. 3. The upper panel includes the complete data set, while the bottom one displays a closer look where the data from the NOT overlap that from Arizona. Also, a synthetic light curve is overplotted (see figure caption for details).

\footnotetext{
${ }^{1}$ Filter details with transmission curves for all NOT filters can be found on http://www. not.iac.es/instruments/filters.

2 Transmission curves for the Montréal 4K CCD camera are found on http://james.as.arizona.edu/ psmith/61inch/FILTERS/ filters.html.
}
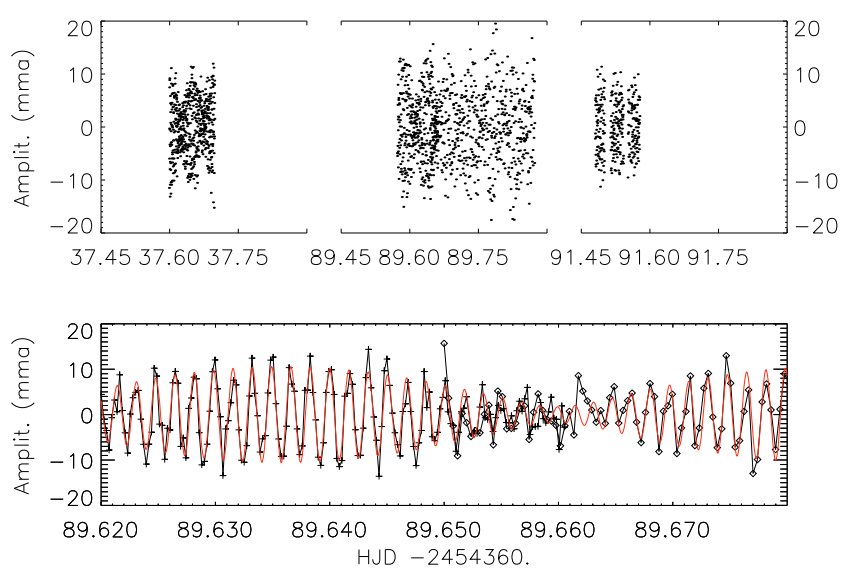

Fig. 3. Light curve of 2M0415+0154. Upper panel: our complete data set. Note the different times for each segment. Bottom panel: detail of the light curve where data from NOT (plus symbols) and from Arizona (diamond symbols) overlap. A synthetic light curve computed from the frequencies obtained (Table 2) is overplotted as a continuous red curve.

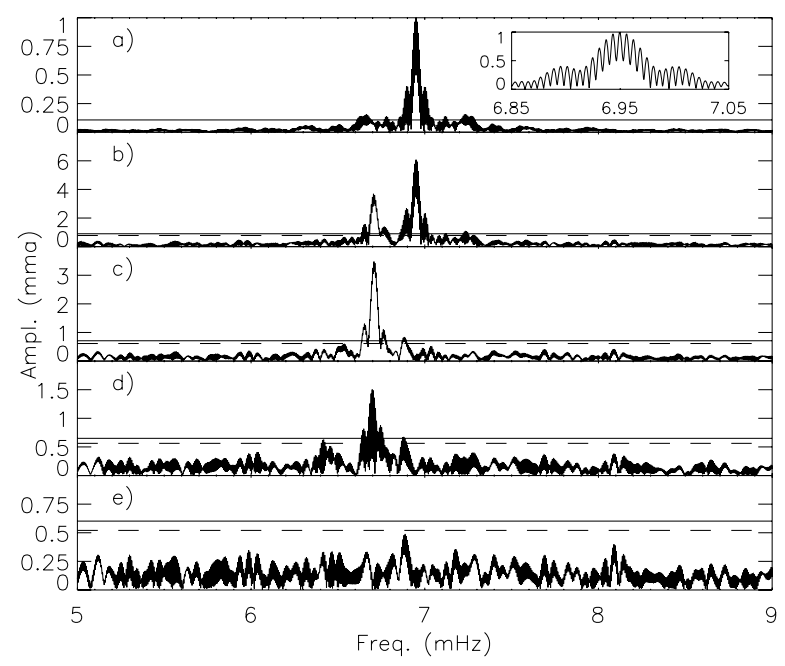

Fig. 4. Frequency analysis of the $B$-filter data set. Panel a): spectral window centered at the highest amplitude frequency. Panel b): amplitude spectrum. Panels c)-e): amplitude spectrum after successive prewhitening of the frequencies in Table 2. Continuous (broken) horizontal lines indicate 3.7 (3.2) times the noise level.

Table 2. Frequencies, amplitudes and phases derived from $B$-band light curve of $2 \mathrm{M} 0415+0154$. Formal fitting errors on the last $\operatorname{digit}(\mathrm{s})$ are included within parentheses.

\begin{tabular}{llccll}
\hline \hline & Freq. $[\mu \mathrm{Hz}]$ & Period [s] & Ampl. [mma] & Phase [rad] & $S / N$ \\
\hline$f_{3}$ & $6703.23(54)$ & 149.28 & $2.0(1)$ & $-0.76(18)$ & 12.7 \\
$f_{2}$ & $6712.23(40)$ & 148.98 & $2.6(1)$ & $-1.32(14)$ & 16.7 \\
$f_{1}$ & $6949.64(5)$ & 143.89 & $6.2(1)$ & $-3.12(2)$ & 40.5 \\
\hline
\end{tabular}

We performed a frequency analysis of the data acquired in December, 2007. This corresponds to the $B$-band photometry obtained both at NOT and Kuiper telescopes during two almost consecutive nights. Panel a) in Fig. 4 includes the spectral window centered at $6.949 \mathrm{mHz}$. Panel b) shows the amplitude spectrum, while other panels display the residual amplitude spectra after successive subtraction of one, two, and three sinusoidal functions from the original light curve. Frequencies, amplitudes and phases obtained from a non-linear least-squares fit are listed in Table 2. Phases are relative to the first data point, 
Table 3. Fit from Winter (2006) and by us to the available spectra of $2 \mathrm{M} 0415+0154$. In parentheses are the $1-\sigma$ fitting errors.

\begin{tabular}{lrrr}
\hline \hline & $T_{\text {eff }}[\mathrm{kK}]$ & $\log g[\mathrm{dex}]$ & $\log \left(N_{\mathrm{He}} / N_{\mathrm{H}}\right)$ \\
\hline Bok 1 $1_{\text {Winter }}$ & $32.88(20)$ & $5.943(35)$ & $-1.390(11)$ \\
Bok 2 $\mathrm{LTE}, 0$ & $33.53(10)$ & $5.87(02)$ & $-1.618(19)$ \\
Bok 2 & $34.03(08)$ & $5.81(02)$ & $-1.584(09)$ \\
Bok 2 NLTE & $34.01(19)$ & $5.79(02)$ & $-1.605(21)$ \\
\hline
\end{tabular}

HJD $=2454449.5720617$. The signal to noise $(S / N)$ of each peak is computed from the residual spectrum after removing this frequency.

The spectral window of this data set has a $F W H M$ of $7 \mu \mathrm{Hz}$, which is adopted as the frequency resolution $(\Delta v)$. This allows $f_{1}$ to be well resolved as an isolated peak. On the other hand, $f_{2}$ and $f_{3}$ are barely resolved, with a frequency distance in between $\Delta v$ and $1 \mathrm{c} / \mathrm{d}$ alias. Although at least two peaks are clearly present at $\sim 6700 \mu \mathrm{Hz}$, their frequencies might not be so accurate as indicated by the formal fitting errors included in Table 2 .

Trying to improve the frequency resolution, the discovery run was also included in the analysis. However, as it is 52 nights apart, the spectral window becomes much more complex. Moreover, a different filter was used for the discovery run $(W)$, which likely produces a light curve with slightly different amplitudes of oscillation. Thus, we prefer the frequency solution from the December run alone listed in Table 2.

\subsection{Spectroscopy}

A single spectrum of $2 \mathrm{M} 0415+0154$ (Fig. 1) was obtained on the night of January 28, 2004 (Bok 1), as part of a large campaign to obtain low resolution spectra of a sample of blue stars selected on the basis of 2MASS colors. This campaign was undertaken using the $\mathrm{B} \& \mathrm{C}$ spectrograph on the University of Arizona $2.3 \mathrm{~m}$ Bok telescope. The physical parameters found by Winter (2006, Appendix C) are listed in Table 3 as Bok $1_{\text {Winter }}$, together with our own values. Due to a setup error, this particular spectrum was obtained with a slit width of 4.5 arcsec instead of the standard 2.5 arcsec. The effective resolution would therefore depend on the seeing during the integration, which can only be roughly estimated. Since the gravity depends strongly on the width of the Balmer lines, the uncertainty in resolution translates directly into a large uncertainty in the line profile fitting. Indeed, the analysis of Winter (2006) assumes the standard FWHM resolution of about $9 \AA$, and we get a result that is lower by 0.2 dex when assuming a resolution of $10 \AA$. However, the true number could be either higher or lower depending on the actual seeing conditions at the time of the observation, and any discrepancy would have a significant impact on the resulting fit.

In order to get a more accurate determination of the physical parameters than was possible from the ambiguous discovery spectrum, and furthermore to check if the star could be a radial velocity variable, new spectroscopy data was acquired after the target was identified as a pulsator.

Four low resolution spectra were obtained with the Bok telescope (see Table 1), this time using the $2.5^{\prime \prime}$ slit. The spectra were processed using standard IRAF tasks. These four spectra were iteratively shifted by cross-correlation against the summed template before finally combining them. The individual spectra have a $S / N$ between 150 and 190, while the combined spectrum achieves a $S / N$ of 350. The presence of many helium and metal lines, and a particularly high $S / N$ produced exceptionally low relative velocity errors considering the low resolution. All crosscorrelation errors are below $15 \mathrm{~km} \mathrm{~s}^{-1}$, which is only $\sim 1 / 35$ of a velocity resolution element. The RV measurements obtained for each four individual spectrum are $-3 \pm 11,-0.5 \pm 14,+6 \pm 15$ and $0.5 \pm 15$ respectively. Since they are all well below the crosscorrelation errors, there is no indication of RV variability at the $15 \mathrm{~km} \mathrm{~s}^{-1}$ level over a three day period.

The combined spectrum (Bok 2) was the subject of a more careful parameter determination than previously allowed. A synthetic grid of metal line blanketed LTE model atmospheres (Heber et al. 1999) was used to derive $T_{\text {eff }}, \log g$ and helium abundance from a simultaneous fit to Balmer and helium lines. The values derived are listed in Table 3 as Bok $2_{\mathrm{LTE}, 0}$, and some line fits are shown in the upper panel of Fig. 5. While a rather good fit is obtained for most of the lines, the core of $\mathrm{H}_{\alpha}$ is not properly reproduced and HeII4686 cannot be matched.

The same problem was encountered by Heber et al. (2006) for the sdB KY Uma (PG 1219+534). Based on the discovery of supersolar abundances of heavy metals in this particular object, the authors succeeded in simultaneously fitting hydrogen and helium lines using a grid of LTE atmospheres with metals scaled by a factor of $10([\mathrm{M} / \mathrm{H}]=1.0)$. This, on the other hand, decreased the discrepancy between temperatures inferred from Balmer line fitting and helium ionization equilibrium. Geier et al. (2007) also faced this helium problem for V2214 Cyg (KPD 1930+2752) which has a similar $T_{\text {eff }}$ as KY Uma and $2 \mathrm{M} 0415+0154$.

Although there is no metal abundance analysis available for $2 \mathrm{M} 0415+0154$, we repeated the line fitting procedure using an LTE model atmospheres grid with enhanced metal content $([\mathrm{M} / \mathrm{H}]=1.0)$. The new parameters obtained are included in Table 3 (Bok $2_{\mathrm{LTE},+1}$ ). The bottom panel of Fig. 5 shows the new line fitting results, note the significantly better match, specially for $\mathrm{H}_{\alpha}$ and HeII4686. Whether this indicates a supersolar heavy elements abundance in 2M0415+0154 should be confirmed with high resolution spectroscopy, although metal abundance analysis by Geier et al. (2008) indicate a temperature-metal abundance correlation.

NLTE effects may also influence the physical parameters determination, as already noticed for other sdBs (e.g. Heber \& Edelmann 2004). For this reason, a new line fit to Bok 2 was produced using a metal free NLTE atmospheres grid. The temperature derived in this case (see Table 3 , Bok 2 NLTE) is slightly higher than the temperature from the LTE grid with solar metallicity, while $\log g_{\mathrm{NLTE}}<\log g_{\mathrm{LTE}, 0}$, as systematically found for other sdB analysis (Heber \& Edelmann 2004). On the other hand, we find no significant difference between the parameters computed using the NLTE grid (Bok 2 NLTE) and the LTE grid with enhanced metallicity (Bok $2_{\mathrm{LTE},+1}$ ). However, we note that the NLTE grid produces the poorest line fit, in particular to $\mathrm{H}_{11}$ and HeII4686, which is reflected in the higher errors in Table 3.

Additionally, two intermediate resolution spectra were obtained at the European Southern Observatory, Chile (ESO), at La Silla using the New Technology Telescope (NTT) equipped with the ESO Multi Mode Instrument (EMMI). Using grating number 3 and a slit width of $1.0^{\prime \prime}$, we obtained a spectral resolution of $\sim 1 \AA$ and a rather modest wavelength coverage of 3880-4380 A. Calibration lamp spectra were taken after every single exposure to ensure reliable radial velocities. Standard procedures from the ESO MIDAS package were used for data reduction.

Whereas the narrow wavelength coverage does not allow reliable physical parameters to be determined, the higher resolution is suitable to check for any radial velocity variation. The 

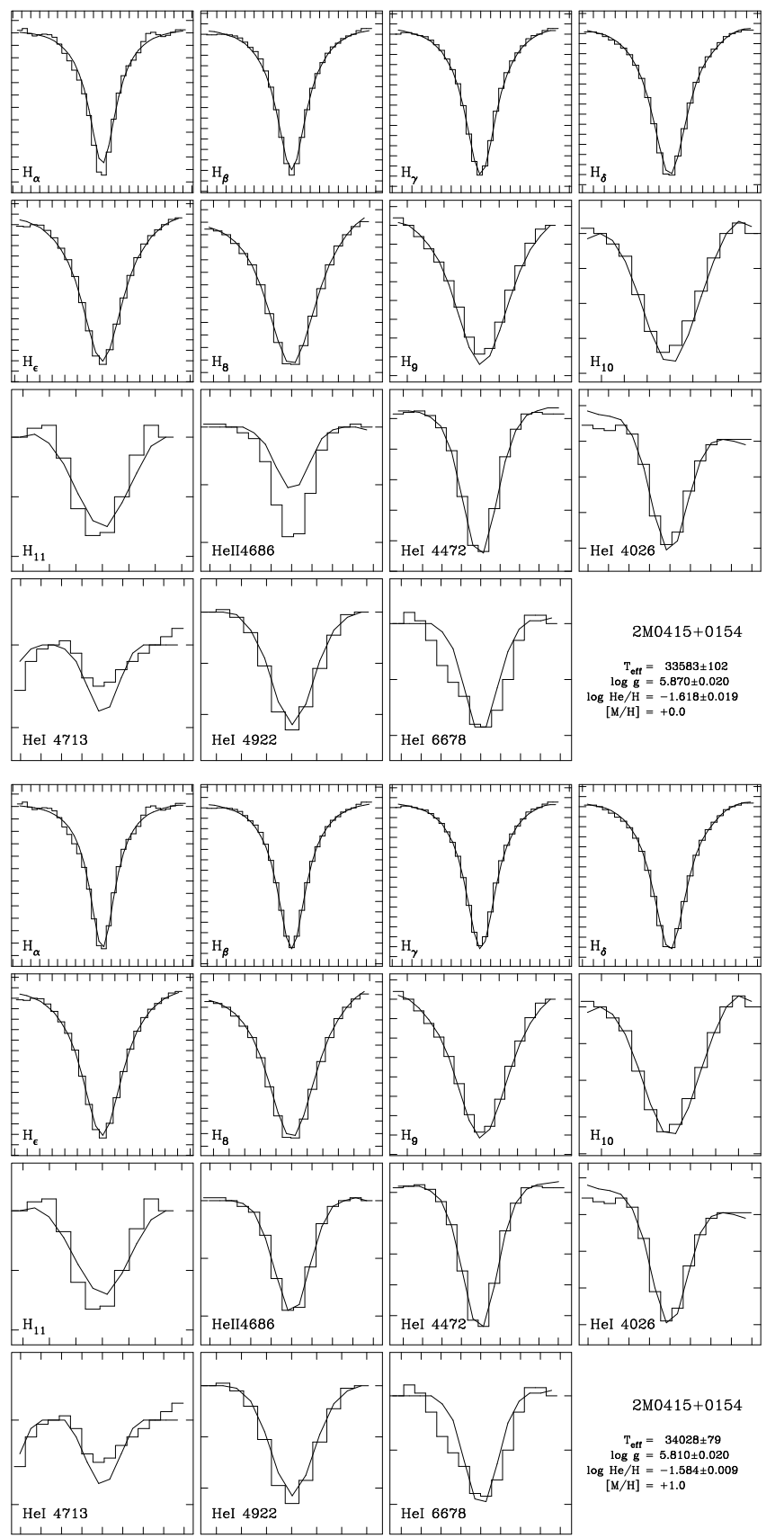

Fig. 5. Fits of model atmospheres to the combined low resolution Bok spectrum of $2 \mathrm{M} 0415+0154$. The upper grid of $4 \times 4$ panels shows the results of fitting a solar metallicity grid to $2 \mathrm{M} 0415+0154$, the lower grid shows the fit when using a super-solar metallicity $([\mathrm{M} / \mathrm{H}]=$ +1.0) grid. In each subpanel the wavelength ticks on the $x$-axis are $5 \AA$ apart and centered on the grids central wavelength of the line. The tics on the $y$-axis are separated by $2 \%$ in flux relative to the continuum.

RV meassurements from the two spectra were determined by calculating the shifts of the measured wavelengths to laboratory data. A simultaneous fit of a set of mathematical functions to the observed line profiles was performed using the FITSB2 routine (Napiwotzki et al. 2004b). We obtained a velocity shift of $10 \pm 13 \mathrm{~km} \mathrm{~s}^{-1}$ and $3 \pm 8 \mathrm{~km} \mathrm{~s}^{-1}$, respectively. Thus, no significant $\mathrm{RV}$ variation is detected between the two observations, separated by $24.1 \mathrm{~h}$, in perfect agreement with the results from the lower resolution observations.
From the three fits we have derived (listed in Table 3), we adopt the following values and for $2 \mathrm{M} 0415+0154: T_{\mathrm{eff}}=34000 \pm 500, \log g=5.80 \pm 0.05$ and $\log \left(N_{\mathrm{He}} / N_{\mathrm{H}}\right)=-1.60 \pm 0.05$.

\section{Conclusions}

We have presented the discovery of a new short period pulsating sdB: 2M0415+0154. A short photometric run revealed two frequencies between 6 and $7 \mathrm{mHz}$ (see Fig. 2). Follow up photometry clearly resolved three frequencies: an isolated $\sim 6$ mmag peak at $6.95 \mathrm{mHz}$ and two relatively close frequencies at $\sim 6.7 \mathrm{mHz}$ of smaller amplitude (see Table 2). The presence of excited modes at the 0.5 mmag level cannot be discarded.

Further spectra of 2M0415+0154 were obtained to check for possible radial velocity variations, and it was concluded that the object is not part a short period binary system, although periods longer than a few days cannot be ruled out. Since the spectra show no signs of a cool companion, as the common sdB +FGK binaries do, 2M0415+0154 must be either single or in a long period $\mathrm{sdB}+\mathrm{WD}$ system. The physical parameters obtained using LTE and NLTE model atmospheres are listed in Table 3. We adopt $T_{\text {eff }}=34000 \pm 500 \mathrm{~K}, \log g=5.80 \pm 0.05 \mathrm{dex}$, $\log \left(N_{\mathrm{He}} / N_{\mathrm{H}}\right)=-1.60 \pm 0.05$ (see Sect. 2.2).

These values place the star well centered within the observed instability region of V361 Hya objects. The periods of oscillation (143-150 s) also agree with the period window of other sdBs of this class. In particular, all pulsating sdBs with similar parameters have also similar frequency spectra: QQ Vir (PG 1325+101), LM Dra (PG 1618+563), LS Dra (HS 1824+5745), KY UMa and V4640 Sgr (EC 20117-4014) have periods in the range $130-160 \mathrm{~s}$, all of them with less than 7 detected modes. UY Sex (PG 1047+003) and EK Psc (PG 0014+067), also with similar $T_{\text {eff }}$ and $\log g$, show 13 peaks in their Fourier spectrum, and thus over a slightly wider period window (80-170 s).

Being fairly bright $(V=13.8) 2 \mathrm{M} 0415+0154$ is ideal for multisite photometric campaigns, and its relatively simple pulsation spectrum makes it a reasonable target for empirical mode identification, either multicolor photometry or time-resolved spectroscopy. This is critically needed in order to constrain the asteroseismic analysis when few frequencies of oscillation are available. The interior structure modeling of 2M0415+0154 would help to understand the disputed formation channels of the apparently single population of sdBs.

Acknowledgements. We thank U. Heber for kindly providing the spectral model grids. R.O. is supported by the Research Council of Leuven University, through grant GOA/2003/04. R.Ø. is supported by the Research Council of the University of Leuven and by the FP6 Coordination Action HELAS of the EU. S.G. was supported by the Deutsche Forschungsgemeinschaft (DFG) under grant He 1354/40-3. E.G. acknowledges the partial support of NASA FUSE grants NNG06GA30G and NNX06AC42G. This publication makes use of data products from the Two Micron All Sky Survey, which is a joint project of the University of Massachusetts and the Infrared Processing and Analysis Center/California Institute of Technology, funded by the National Aeronautics and Space Administration and the National Science Foundation.

\section{References}

Ahmad, A., \& Jeffery, C. S. 2005, A\&A, 437, L51

Alvarez, M., Hernandez, M. M., Michel, E., et al. 1998, A\&A, 340, 149

Charpinet, S., Fontaine, G., Brassard, P., et al. 1997, ApJ, 483, L123

Christensen-Dalsgaard, J. 2002, Rev. Mod. Phys., 74, 1073

Dorman, B., Rood, R. T., \& O'Connell, R. W. 1993, ApJ, 419, 596 
Fontaine, G., Brassard, P., Charpinet, S., et al. 2003, ApJ, 597, 518

Geier, S., Nesslinger, S., Heber, U., et al. 2007, A\&A, 464, 299

Geier, S., Heber, U., \& Napiwotzki, R. 2008, in Hot Subdwarf Stars and Related Objects, ed. U. Heber, C. S. Jeffery, \& R. Napiwotzki, ASP Conf. Ser., 392, 159

Green, E. M., Liebert, J. W., \& Saffer, R. A. 1997, in The Third Conference on Faint Blue Stars, ed. A. G. D. Philip, J. Liebert, R. Saffer, \& D. S. Hayes, 417 Green, E. M., Fontaine, G., Reed, M. D., et al. 2003, ApJ, 583, L31

Green, E. M., Fontaine, G., Hyde, E. A., For, B.-Q., \& Chayer, P. 2008, in ASP Conf. Ser. 392, ed. U. Heber, C. S. Jeffery, \& R. Napiwotzki, 75

Han, Z., Podsiadlowski, P., Maxted, P. F. L., Marsh, T. R., \& Ivanova, N. 2002, MNRAS, 336, 449

Han, Z., Podsiadlowski, P., Maxted, P. F. L., \& Marsh, T. R. 2003, MNRAS, 341, 669

Heber, U. 1986, A\&A, 155, 33

Heber, U., \& Edelmann, H. 2004, Ap\&SS, 291, 341

Heber, U., Reid, I. N., \& Werner, K. 1999, A\&A, 348, L25

Heber, U., Hirsch, H., Ströer, A., et al. 2006, Baltic Astron., 15, 91

Hu, H., Dupret, M.-A., Aerts, C., et al. 2008, A\&A, 490, 243

Jeffery, C. S., \& Saio, H. 2006, MNRAS, 372, L48
Kilkenny, D., Koen, C., O’Donoghue, D., \& Stobie, R. S. 1997, MNRAS, 285, 640

Maxted, P. F. L., Heber, U., Marsh, T. R., \& North, R. C. 2001, MNRAS, 326, 1391

Napiwotzki, R., Karl, C. A., Lisker, T., et al. 2004a, Ap\&SS, 291, 321

Napiwotzki, R., Yungelson, L., Nelemans, G., et al. 2004b, in Spectroscopically and Spatially Resolving the Components of the Close Binary Stars, ed. R. W. Hilditch, H. Hensberge, \& K. Pavlovski, ASP Conf. Ser., 318, 402

Østensen, R., \& Solheim, J.-E. 2000, Baltic Astron., 9, 411

Østensen, R., Heber, U., Silvotti, R., et al. 2001, A\&A, 378, 466

Rodríguez-López, C., Ulla, A., \& Garrido, R. 2007, MNRAS, 379, 1123

Schuh, S., Huber, J., Dreizler, S., et al. 2006, A\&A, 445, L31

Skrutskie, M. F., Cutri, R. M., Stiening, R., et al. 2006, AJ, 131, 1163

Stroeer, A., Heber, U., Lisker, T., et al. 2007, A\&A, 462, 269

Winget, D. E., \& Kepler, S. O. 2008, ARA\&A, 46, 157

Winter, C., Jeffery, C. S., Ahmad, A., \& Morgan, D. R. 2006, Baltic Astron., 15, 69

Woudt, P. A., Kilkenny, D., Zietsman, E., et al. 2006, MNRAS, 371, 1497

Zacharias, N., Monet, D. G., Levine, S. E., et al. 2005, VizieR Online Data Catalog, 1297, 0 